\title{
Effects of Geometry in Atrial Fibrillation Markers Obtained With Electrocardiographic Imaging
}

\author{
Rubén Molero ${ }^{1}$, Andreu M Climent ${ }^{2}$, Ismael Hernández-Romero ${ }^{2}$, Alejandro Liberos ${ }^{2}$, Francisco \\ Fernández-Avilés ${ }^{2}$, Felipe Atienza ${ }^{2}$, María S Guillem ${ }^{1}$, Miguel Rodrigo ${ }^{1}$ \\ ${ }^{1}$ ITACA Institute, Universitat Politècnica de València, Valencia, Spain, \\ ${ }^{2}$ Cardiology Department, Hospital GU Gregorio Marañón, IiSGM, CIBERCV, Spain
}

\begin{abstract}
Electrocardiographic imaging (ECGI) can characterise cardiac pathologies such as atrial fibrillation $(A F)$ through specific markers based on frequency or phase analysis. In this study, the effect of the geometry of patients' torso and atria in the ECGI resolution is studied.

A realistic $3 D$ atrial geometry was located on 30 patient torsos and ECGI signals were calculated for 30 different $A F$ simulations in each torso. Dominant frequency $(D F)$ and reentrant activity analysis were calculated for each scenario. Anatomical and geometrical measurements of each torso (30-80\% of variability between patients) and atria were calculated and compared with the errors in the ECGI estimation versus the departing EGM maps.

Results show evidences that big chest dimensions worsen the non-invasive calculation of $A F$ markers $(p<0.05)$. Also, higher number of visible electrodes from each atrial region improves ECGI characterization measured as lower DF deviations $(0.64 \pm 0.26 \mathrm{~Hz}$ vs $0.72 \pm 0.27 \mathrm{~Hz}, \quad p<0.05)$ and higher reentrant activity coincidence $(10.1 \pm 12.2 \%$ vs $3.4 \pm 3.4 \%, p<0.05)$.

Torso and atrial geometry affect the quality of the noninvasive reconstruction of $A F$ markers such as $D F$ or reentrant activity. Knowing the geometrical parameters that worsen non-invasive AF maps may help to measure each detected $A F$ driver reliability.
\end{abstract}

\section{Introduction}

Atrial fibrillation (AF) is the most common heart disease whose main therapies, both pharmacological and surgical, provide insufficient outcomes [1]. Detecting and electrically isolating the atrial regions whose electrical activity plays a dominant role in maintaining the arrhythmia can benefit the ablation strategies. These dominant regions are nowadays identified using intracardiac catheters, which detect higher activation rates through dominant frequency (DF) analysis, or atrial regions with presence of reentrant activity, through phase analysis.

Electrocardiographic imaging (ECGI) is a non-invasive technique that uses the 3D patient geometry and signals registered with Body Surface Potential Mapping (BSPM) to reconstruct the electrical activity of the cardiac epicardium [2]. ECGI brings anatomically located electrical information and is used to detect AF-driving regions using also DF and phase analysis [3].

The anatomy of the patient and the torso inhomogeneities affect the ECGI reconstruction accuracy [4], which may hinders the proper AF characterization with ECGI. In this work, the effect of the anatomical characteristics of patients in the ECGI reconstruction is studied. We will measure how the geometry of the atria and patients' torso can affect to the quality of the non-invasive reconstruction of $\mathrm{AF}$ markers used to guide ablation procedures.

\section{Materials and Methods}

\subsection{Data acquisition}

MRI/CT images from 30 patients (23 females and 7 males; $61 \pm 14$ years) with $\mathrm{AF}$ were acquired 2-3 days prior to the ablation procedure. Atria and torso anatomy were obtained by segmentation of MRI images by using ITKSNAP [5]. Additionally, the torso anatomy, together with the surface electrode location was obtained by photogrammetry techniques. Photographic images from multiple points of view were obtained for each patient wearing the recording electrodes. Posteriorly, these images were processed by photogrammetry in order to reconstruct the torso anatomy with the surface electrode positions. Atria and torso anatomies from MRI/CT and photogrammetry were co-localized using rigid transformations based on manually-annotated landmarks. Photogrammetry-obtained torso was used to the torso analysis, whereas the MRI anatomy was used to colocalize the atrial and torso geometry. 


\subsection{Mathematical models}

A realistic atrial $3 \mathrm{D}$ model was used to simulate the electrical activity during AF episodes. Modifications in the ionic currents and fibrosis were introduced in the model to create 30 simulations of AF maintained by functional reentries. For each simulation, the equivalent electrogram (EGM) was calculated in the epicardial surface of the model [2]. The ECG potentials were obtained solving the forward problem, and the inverse-computed electrogram (icEGM) was solved by calculating the inverse problem using zero-order Tikhonov and L-curve methods [2].

For each patient, the simulated geometrical atrial model was positioned inside the torso coinciding with the natural atrial location. ECG and icEGM signals were then calculated for the 30 simulations and 30 torsos (Figure 1.A). The icEGMs were calculated for two scenarios, using the information of 256 and 64 electrodes in the torso surface. For each scenario, two white Gaussian noise levels were added to the surface ECG signals, $30 \mathrm{~dB}$ (low noise) and $10 \mathrm{~dB}$ (high noise). To compare the results of the ECGI reconstruction, dominant frequencies (DF) and reentrant activity analysis of the icEGM and EGM signals were computed. To obtain the DF maps, the power spectral density was calculated using Welch periodograms (Fig. 1.B). Hilbert transform was used to identify singularity points (SPs) as previously described (Fig. 1.C) [2].

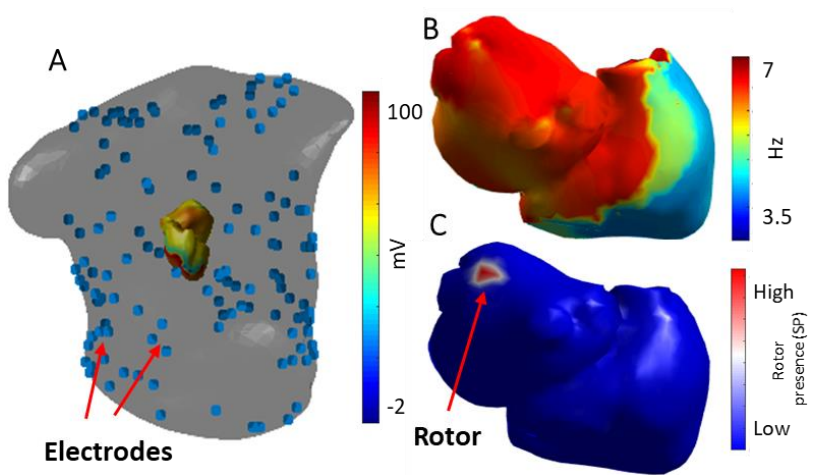

Figure 1. (A) Representation of the torso of a patient covered with 64 electrodes with an adjusted atrial model; (B) Dominant Frequency map (C) and Singularity Point representation of non-invasively reconstructed ECGI signals.

\subsection{Geometrical measurements}

In order to identify which anatomical parameters affect the quality of the ECGI reconstruction, each patient's torso was measured. Global anatomy measurements such as torso surface and volume were calculated. Torso measurements were separated in the chest and abdomen sections, to identify each area separately. For both areas, the maximum area and perimeter, and the diameter in the coronal (x) and sagittal (y) plane were calculated.
Moreover, for each patient, the minimum distance from the atria epicardium and the torso was calculated.

For each anatomical measurement, patients were classified in two groups, depending on the measured parameter (high or low). The distinction between high and low values was made by sorting the 30 patients' measurements from lowest to highest value and separating them into two groups with significant differences in the measured parameter. Group separation was within $50-50 \%$ and $40-60 \%$ proportions.

Finally, the number of visible electrodes from each atrial node was calculated for the 256 and 64 electrodes cases. This measurement represents the number of electrodes that can be directly seen from each vertex of the atria model with no interposition of other anatomical obstacles. These surface electrodes are supposed to provide direct electrical information to reconstruct the ECGI signals.

\subsection{Error measurements}

DF maps and SPs histograms of the icEGM were compared with the departing DF maps and SPs histograms obtained with the EGM signals, in order to quantify the quality of the ECGI reconstruction. Absolute and relative deviations on each individual DF value were calculated, as well as the deviation on the Highest DF calculation. The singularity points' histograms were compared calculating distances between sites with maximal reentrant activity (SP presence). Also, a comparison point by point of the SP spatial histogram was calculated (SP global coincidence), and the comparison of the regions with high SP presence (SP>50, SP coincidence). Non-paired t-student test was performed to assess significant differences between measures.

\section{Results}

\subsection{Anatomical influence in ECGI}

Different anatomical parameters were evaluated in 30 patients' torsos to assess how they can affect in the quality of the ECGI reconstruction. In Figure 2, the p-values of each parameter are presented for the worst-case scenario calculated, 64 electrodes and $10 \mathrm{~dB}$ of noise. In each case, the different AF marker measures (columns) were tested between the 2 groups with higher and lower anatomical parameters (rows). The parameters that have statistical significance in affecting the resolution of the IP are the ones related with the size of the chest of the patient. The higher values of the maximum chest surface, perimeter and diameter in the coronal plane, the higher deviation in the ECGI reconstruction. The minimum distance from atria to torso was also strongly related with the quality of the ECGI resolution. However, there was not statistical evidence that 
a higher abdomen size can worsen the quality of the ECGI reconstruction in most of the AF measures.

Markers presenting more statistical significance in the quality of the ECGI reconstruction against anatomical deviations were the absolute DF error, and the global coincidence of DFs and SPs.

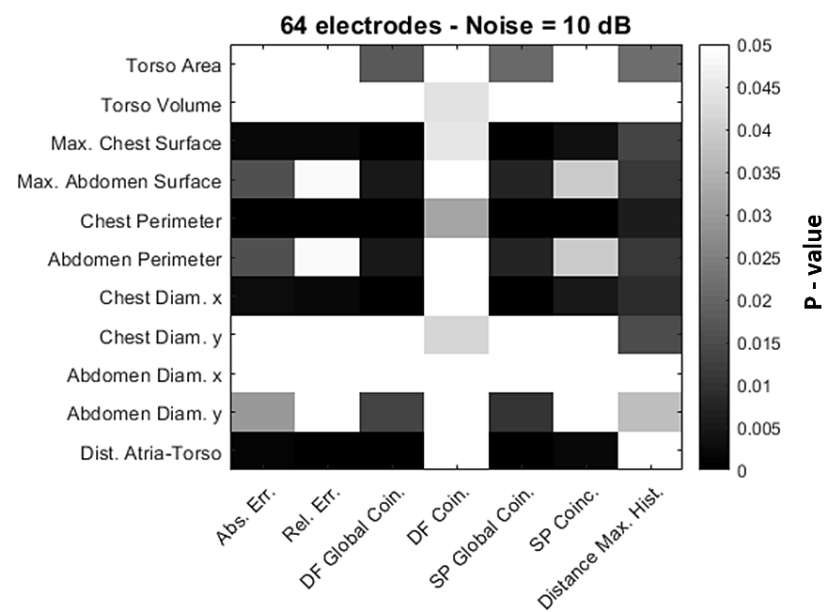

Figure 2. P-values of different atrial fibrillation marker measures (columns) tested between the 2 patient groups with higher and lower values of anatomical parameters (rows), using 64 electrodes and applying $10 \mathrm{~dB}$ of noise.

\subsection{ECGI accuracy on atrial locations}

In Figure 3.A the DF map from the departing EGM signals is shown, where the red dashed line shows the atrial region area in which the number of visible surface electrodes was higher, over the percentile 33. This threshold was used as a subjective criterion because of differences between groups were more noticeable. Figure 3.B shows the DF map obtained from ECGI for the same AF simulation. It can be observed that the area out of the dashed line has more differences between EGM and ECGI maps than the area with higher number of visible electrodes. The absolute error in DF for the 30 patients is presented in Figures 3.C and 3.D. These figures show that for higher values of visible electrodes, the absolute error in the DF icEGM is lower, e.g. $0.64 \pm 0.26 \mathrm{~Hz}$ vs $0.72 \pm 0.27$ $\mathrm{Hz}(\mathrm{p}<0.01)$ respectively, for 256 electrodes and $30 \mathrm{~dB}$ of noise. Besides, it is shown that for higher number of electrodes used for the calculation (256) and lower noise introduced $(30 \mathrm{~dB})$ the absolute error is lower, $0.64 \pm 0.26$ $\mathrm{Hz}$, than for the opposite case, $0.82 \pm 0.25 \mathrm{~Hz}(\mathrm{p}<0.01)$.

Figures 4.A and 4.B show the rotor presence in EGM and ECGI maps respectively. The red dashed line shows again the atrial regions with more/less than the $33 \%$ of surface electrodes visible. It can be noticed that there is a higher similarity in the reconstruction of the area with a higher number of electrodes visible (lower area of the atria). Figures 4.C and 4.D show the percentages of SP coincidence between the atria model for the $30 \mathrm{AF}$ simulations and the 30 patients' torsos. In the cases in which we introduce less noise $(30 \mathrm{~dB})$, a greater number of visible electrodes from the atria increases the percentage of coincidence between the EGM and ECGI reentrant maps: $10.11 \pm 12.2 \%$ vs $3.37 \pm 3.4 \%$ using 256 electrodes, $(\mathrm{p}<0.05)$ and $7.5 \pm 10.5 \%$ vs $1.73 \pm 1.1 \%$ using 64 electrodes, $(\mathrm{p}<0.05)$. In addition, as Figures 4.C and 4.D show, the higher the noise and the lower number of electrodes, the greater the error observed in the ECGI calculations. No statistically significant evidence was found for the high noise scenario ( $p>0.05)$, although the ECGI reconstruction was higher when more surface electrodes were visible from the atria.

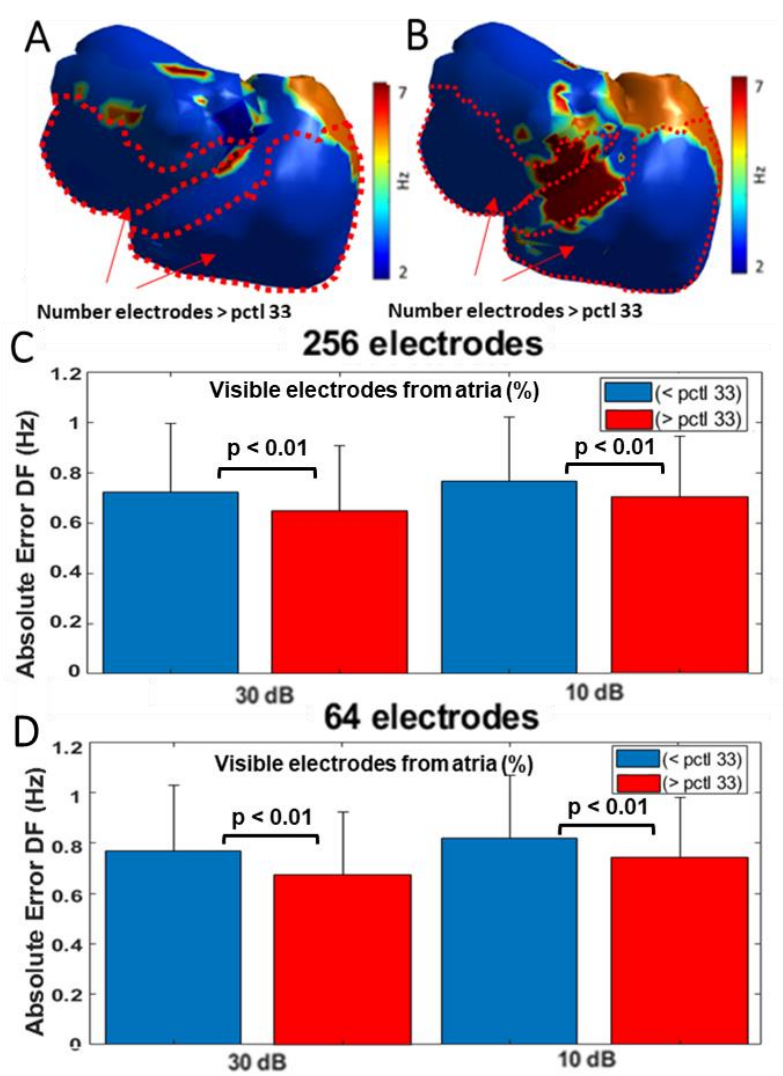

Figure 3. (A) Dominant frequency (DF) map for departing EGM signals (A) and non-invasively reconstructed ECGI signals (B). Red dashed line represents the atrial regions whose number of visible electrodes is over the percentile 33 (pctl 33). (C) Absolute DF error between original EGM and ECGI maps for atrial regions with low/high number (pctl 33) of visible electrodes $(\mathrm{N}=900)$ using 256 and (D) 64 electrodes.

\section{Discussion}

Several studies report the effect of the different torso heterogeneities in the ECGI reconstruction $[4,6]$, but the effect of the geometry of the torso in IP solution has not been reported. In this work, we showed that anatomical 


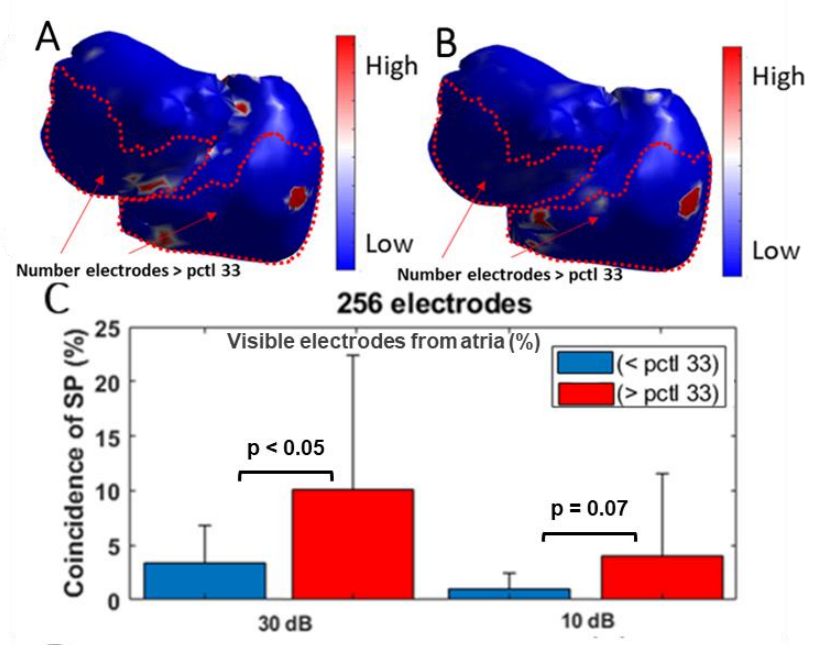

D

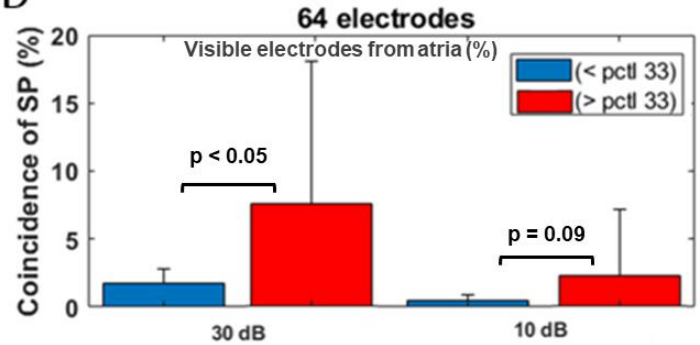

Figure 4. Singularity point (SP) map for departing EGM signals (A) and non-invasively reconstructed ECGI signals (B). Red dashed line represents the atrial regions whose number of visible electrodes is over the percentile 33 (pctl 33). (C) Coincidence of SP detection between EGM and ECGI maps for atrial regions with low/high number (pctl 33 ) of visible electrodes ( $N=900)$ using 256 and (D) 64 electrodes.

characteristics of the patients can affect the resolution of the ECGI. It was expected that in patients with higher body dimensions, the ECGI maps may be worse due to interferences of the amount of fat. It has been shown that greater chest dimensions worsen the ECGI calculation, nevertheless, an abdomen with large dimensions did not affect that much in its resolution. The distance from the epicardium of the atria to the torso would also influence the calculation of the inverse problem: the closer the atrial anatomy to the surface electrodes, the better the quality of the ECGI reconstructed signals.

The number of visible surface electrodes from the atria is a parameter that indicates the amount of information that directly arrives to the atria through the torso to solve the inverse solution. It was expected that the greater number of visible electrodes in each vertex of the atria model, the better resolution of these problems as more information is available to perform the calculation. For the DF errors and of the SP histogram, evidences that the higher the number, the better the ECGI result were found.

In this study, only a mathematical model of the atria was used with 30 simulations of AF. In further studies, different atria anatomies should be used with real AF signals.

\section{Conclusion}

The identification of geometrical and anatomical parameters that can lead to an inaccurate ECGI reconstruction can be useful to create maps with higher concordance with the epicardial activity. Patients with bigger chest dimensions have been showed to worsen the ECGI quality. Although big chest dimensions worsen the IP quality, the error due to the geometry is not high, due to that, the results of this study should be compared with calculations with real atria and signals from patients.

\section{Acknowledgements}

Supported in part by: Instituto de Salud Carlos III FEDER (Fondo Europeo de Desarrollo Regional; IJCI2014-22178, DTS16/00160; PI14/00857, PI16/01123; PI17/01059; PI17/01106), Generalitat Valenciana Grants (APOSTD/2017 and APOSTD/2018) and projects (GVA/2018/103) and EIT-Health 19600 AFFINE.

\section{References}

[1]S. Narayan et al., "direct or coincidental elimination of stable rotors or focal sources may explain successful atrial fibrillation ablation," Journal of the American College of Cardiology, vol. 62, no. 2, pp. 138-147, July 2013.

[2]M. Rodrigo et al., "Highest dominant frequency and rotor positions are robust markers of driver location during noninvasive mapping of atrial fibrillation: A computational study," Heart Rhythm, vol. 14, no. 8, pp. 1224-1233, Aug. 2017. [3]J. Pedrón-Torrecilla et al. "noninvasive estimation of epicardial dominant high-frequency regions during atrial fibrillation," Journal of Cardiov. Electrophys., vol. 27, no. 4, pp. 435-442, Jan. 2016.

[4]C. Ramanathan et al., "electrocardiographic imaging: i. effect of torso inhomogeneities on body surface electrocardiographic potentials," Journal of Cardiov. Electrophys. vol. 12, no. 2, pp. 229-240, Feb. 2001.

[5]P. Yushkevich et al., "Continuous medial representation for anatomical structures," IEEE Trans. Med. Imaging, vol. 25, no. 12, pp. 1547-64, Dec. 2006.

[6]N. Zemzemi et al., "Effect of the torso conductivity heterogeneities on the ECGI inverse problem solution," Computing in Cardiology, vol. 42, pp. 233-236, Sept. 2015.

Address for correspondence:

Rubén Molero Alabau.

ITACA. Edificio 8G acceso B. Universitat Politécnica de València. Camino de Vera s/n. 46022 Valencia, Spain. rumoal1@upvnet.upv.es 